\title{
Editorial for Weed Science, Volume 62
}

\author{
William K. Vencill*
}

Weed Science continues to be one of the premier journals in the discipline and in the agronomic sciences. The Editorial Board and staff strive to improve the efficiency of the publication process and improve the scope of the journal. Weed Science is seeking to publish more reviews and symposium articles. This year, we will publish a symposium on the environmental effects of herbicide resistance. Work is progressing on a special issue that will serve as a research methods book.

There will be a couple of changes to the journal format for 2014. First, you will notice that the journal has a new literature citation style. This style is more streamlined and similar to what many journals are doing now.

The other change may not be as noticeable, but Weed Science is now using the ORCID (open researcher and contributor ID) system. Authors' ORCID numbers will be a part of the footnote that identifies each author's affiliation (university, business, etc.). The ORCID is a unique 16-digit number that identifies separate authors when they have similar names or initials. It is similar to a DOI (digital object identifier), but it identifies authors instead of articles. If you are not familiar with ORCIDs, more information can be found at http:// orcid.org/. PeerTrack has been modified so that authors can provide their ORCIDs when they submit a manuscript.

The excellent reputation of the journal would not be possible without the cutting-edge research submitted by authors. For the 12 mo preceding September 1, 2013, 211 manuscripts were submitted to Weed Science, compared with 210 in the previous 12 -mo period. Of the manuscripts submitted, 41\% were accepted, compared with 49\% the previous year. The time from submission to first decision was $47 \mathrm{~d}$ this past year compared with $58 \mathrm{~d}$ for the previous year, and the time from submission to final decision was $107 \mathrm{~d}$. Table 1 shows the Impact Factor for Weed Science and other related journals for 2011 and the aggregated numbers during the past $5 \mathrm{yr}$.

\footnotetext{
DOI: $10.1614 /$ editorial-62.1.1

* Professor and Editor, Department of Crop and Soil Sciences, University of Georgia, Athens, GA 30602. Corresponding author's E-mail: wvencill@uga.edu
}

Table 1. Impact factor of Weed Science and comparable journals in 2011 (Anonymous 2013).

\begin{tabular}{lcc}
\hline Journal & $\begin{array}{c}2011 \text { impact } \\
\text { factor }\end{array}$ & $\begin{array}{c}\text { 5-yr impact } \\
\text { factor }\end{array}$ \\
\hline Weed Science & 1.759 & 1.770 \\
Weed Research & 2.045 & 1.994 \\
Crop Protection & 1.303 & 1.598 \\
Pest Management Science & 2.594 & 2.771 \\
Agronomy Journal & 1.518 & 1.989 \\
\hline
\end{tabular}

For the year preceding September 1, 2013, the journal depended on 312 unique reviewers from 30 countries for peer review. We genuinely appreciate the diligent work of the Editorial Board and the hundreds of refereed reviews that are submitted annually. I personally wish to thank the members of the Editorial Board and reviewers for all their efforts on behalf of the journal in 2013 .

Editorial Board 2013

Wun S. Chao

Sharon Clay

Adam Davis

Franck E. Dayan

J. Anita Dille

William E. Dyer

Timothy L. Grey

Amy Lawton-Rauh

John L. Lindquist

Stephen Murphy

Vijay Nandula

Chris Preston

Dean Riechers

Hilary Sandler

Steven Seefeldt

Patrick J. Tranel

Theodore Webster

Martin M. Williams II

Weed Science Reviewers, November 16, 2012November 15, 2013

Ana Alonso-Simón

James V. Anderson

Randy L. Anderson

Christian Andreasen

Andre Andres 
Renee S. Arias

Jamshid Ashigh

Muthukumar V. Bagavathiannan

Michael Barrett

Carol Baskin

Lammert Bastiaans

Daizy Rani Batish

Hugh J. Beckie

Roberto Benech-Arnold

Diane Lyse Benoit

Lori K. Benoit

Brian L. Beres

Mark Lee Bernards

Henrique von Hertwig Bittencourt

Robert E. Blackshaw

Victoria Borowicz

Daniel Brainard

James T. Brosnan

Ian C. Burke

James D. Burton

Philip Busey

Roberto Busi

Ana Caicedo

John Cardina

Leonardo Bianco Carvalho

Demosthenis Chachalis

Rakesh S. Chandran

Raghavan Charudattan

Bhagirath Singh Chauhan

Michael J. Christoffers

Sharon Clay

Warren Conway

Daniel Cook

Miguel Copete

Roberto J. Crespo

Cristina Cruz

Hugo Cruz-Hipolito

William Stevenson Curran

Caleb D. Dalley

Christos Damalas

Henri Darmency

Avishek Datta

Vince M. Davis

Rafael Alberto De Prado Amian

Chantal Dechamp

Carla Andréa Delatorre

Jeffrey F. Derr

J. Anita Dille

Giovanni Dinelli

Antonio DiTommaso

Douglas J. Doohan

Stephen Duke

John Franklin Egan
Ilias George Eleftherohorinos

Norman C. Ellstrand

Seyed V. Eslami

Paul C.C. Feng

Jason Ferrell

Rodrigo Figueroa

Albert Fischer

Frank Forcella

George Frisvold

Todd A. Gaines

Eric R. Gallandt

Travis W. Gannon

David Gealy

Roland Gerhards

Kevin D. Gibson

Les Glasgow

Greta Gramig

Timothy L. Grey

James L. Griffin

Robert H. Gulden

Linda Hall

Bradley D. Hanson

Stuart Patrick Hardegree

K. Neil Harker

Kent Kent Harrison

Jeffrey Herrick

Melissa J. Hills

Robert E. Hoagland

David P. Horvath

Jared Adam Hoyle

Cynthia Huebner

Andrew G. Hulting

Lynn Ingegneri

Claudia S. Ingham

Jordi Izquierdo

Marie A. Jasieniuk

Katherine M. Jennings

Prashant Jha

William G. Johnson

Nicholas Royal Jordan

Shiv Kaundun

George Ogada Kegode

Andrew Robert Kniss

Klaus Kreuz

Per Kudsk

Virender Kumar

Fabiane Pinto Lamego

Sarah Lancaster

Lawrence W. Lass

Ryan Lee

Ramon G. Leon

Matt Liebman

Ginger G. Light

2 - Weed Science 62, January-March 2014 
John L. Lindquist

Rick S. Llewellyn

Christopher John Lortie

Jon Lundgren

Mark Lundy

H.S. Lur

Gregory MacDonald

Gulshan Mahajan

Carol A. Mallory-Smith

Joseph H. Massey

Mitsuhiro Matsuo

Bruce D. Maxwell

Lambert B. McCarty

William B. McCloskey

Patrick E. McCullough

Joseph Scott McElroy

Kristen E. McNaughton

Fabian Daniel Menalled

Lindsey Milbrath

Steven B. Mirsky

Charles L. Mohler

Martin Mortimer

Thomas C. Mueller

Paul Neve

Robert L. Nichols

Scott Jay Nissen

Dennis Calvin Odero

Maria Dolores Osuna Ruiz

Micheal D. K. Owen

William Louis Patzoldt

Alejandro Perez-Jones

Dallas E. Peterson

Angela R. Post

Andrew Jennings Price

Atul Puri

Alan Raybould

Jordi Recasens

Emilie E. Regnier

Karen A. Renner

Boris Rewald

Darren E. Robinson

Frank Rossi

Matthew Ryan

Robert Douglas Sammons

Brian J. Schutte

Julio Alejandro Scursoni

Brent A. Sellers
Dale L. Shaner

David R. Shaw

Anil Shrestha

Peter H. Sikkema

Samunder Singh

Reid J. Smeda

Upender R. Somireddy

Lynn M. Sosnoskie

Josef Soukup

Christy L. Sprague

Phillip Stahlman

Rex Stanton

Tracy M. Sterling

Neal Stewart

David E. Stoltenberg

Stephen D. Strachan

Clarence J. Swanton

Andreu Taberner

François J. Tardif

John Teasdale

Ilias S. Travlos

Mahesh K. Upadhyaya

Rene Van Acker

Mark VanGessel

Martin Miguel Vila-Aiub

Jean Wagner

JinXin Wang

Sarah Ward

Shouhui Wei

Jeff Weidenhamer

Martin Weis

Paula Westerman

Leslie Ann Weston

Philip Westra

James H. Westwood

Timothy Lee Widmer

Hanwen Wu

Marcos Ezequiel Yanniccari

Bryan G. Young

Stephen L. Young

Bernard H. Zandstra

Lewis H. Ziska

\section{Literature Cited}

Anonymous (2013) 2011 Journal Citation Reports: Science Edition 2013. New York: Thomas Reuters 